\title{
Relativistic diffusion of quarks in random gluon fields
}

\author{
Z. Haba \\ Institute of Theoretical Physics, University of Wroclaw, \\ 50-204 Wroclaw, Plac Maxa Borna 9, Poland \\ email:zhab@ift.uni.wroc.pl
}

\begin{abstract}
We consider Wong equations for a particle with a continuous mass spectrum in a random Yang-Mills field approximating the quantum field at finite temperature. We show that particle time evolution can be approximated by a relativistic diffusion. Kubo's generator of the relativistic diffusion is defined as an expectation value of the square of the Liouville operator.
\end{abstract}

\section{Introduction}

The diffusion approximation is well-known in non-relativistic physics [1]. It has been applied in a description of the quark-gluon plasma and in heavy ion collisions [2][3][4][5]. If QCD is considered as the fundamental theory of strong interactions then the diffusion should result from the QCD transport theory [6][7][8]. The form of the diffusion operator can in principle be determined on the basis of the Boltzmann kinetic equation after a calculation of the scattering amplitude [3][9]. A semi-classical approach to the transport theory based on the Wong equation [10] ( derived from quantum dynamics in [11][12][13]) leads to an analog of the Vlasov equation [6][7] [13][14]. Quantized gauge fields in the Vlasov equation have been discussed in [15][16][17]. These authors have shown that quark's time evolution in a quantum gauge field leads to a diffusion of momentum and color. However, the generator of the diffusion has not been defined in an unambiguous way ( the coefficients of the diffusion equation are undetermined and the relativistic invariance remains obscure). It is well-known [18][19] that the Vlasov equation describing a tracer particle moving in plasma in a random electromagnetic field produced by a chaotic motion of other particles can be approximated by a diffusion equation. The generator of the diffusion has been calculated in [18][19]. A detailed discussion of the diffusion approximation to a random non-relativistic dynamics can be found in [20][21] and [22]. 
There is no consensus on the relativistic form of the diffusion (see the reviews in [23][24]). A relativistic diffusion in the configuration space does not exist [25][26]. If we assume that the diffusing particle has a fixed mass then an analog of the Kramers diffusion (in momentum space) is uniquely defined by its generator being the Laplace-Beltrami operator on the mass-shell as shown by Schay [27] and Dudley [28] (for further developments in this approach see [29][30]). There are some other suggestions for a definition of the relativistic diffusion without the assumption that the diffusion takes place on the massshell [23][24][31].

In this paper we start from the basic principles of quantum gauge theory at finite temperature. We assume the gauge and Lorentz covariance in the most general form. It is known that the thermodynamic equilibrium is defined in the rest frame. Then, if the finite temperature theory is Lorentz covariant then it can be transformed to an arbitrary Lorentz frame described by a unit timelike four-vector $w^{\mu}$. We consider the two-point function of gauge covariant field strength [32][33] $\hat{F}_{\mu \nu}(x)$. We show that the generator of the diffusion of momentum depends only on the form of the two-point function of $\hat{F}_{\mu \nu}$ at coinciding points. The diffusion tensor can be related to the energy-momentum tensor of gluons in the thermal state. We do not assume that the particles (quarks) are on the mass-shell as we did in the case of (zero-temperature) QED in [34]. Only free stable particles satisfy the mass-shell condition. Relaxing this condition allows an explicit use of the Lorentz covariance ( four independent components of the momentum;the transport theory for particles with a continuous mass spectrum has been discussed also in ref.[35]). In this paper we extend the covariant calculation of the diffusion in momentum space derived for QED at finite temperature in [36] to QCD. For this purpose we apply the Wong approximation for quark dynamics in QCD. The plan of the paper is the following. In sec.2 we formulate the Wong equation in the random field. In sec.3 we calculate the expectation value of the random evolution (the square of the Liouville operator) which determines the diffusion generator. We discuss the resulting diffusion equation and its applications.

\section{Kinetic equation of Wong dynamics in a Yang- Mills field}

We consider a classical approximation to quantum gauge theory when quark evolution is described by classical trajectories and gluons are approximated by classical Yang-Mills fields. Such an approximation can be justified in the regime of low external momenta and high temperature. In this regime the approximation leads to the Wong equation [10] which can be derived from QCD quantum dynamics [11][12][14].

In Wong dynamics we have an extended phase space: a product $\mathcal{P} \times R^{n}$ 
of the usual phase space $\mathcal{P}$ of position and momenta $(x(\tau), p(\tau))$ and the color described by $n$ coordinates $Q^{a}$. The evolution parameter $\tau$ is chosen as proportional to the proper time. The dynamics of a particle in a Yang-Mills field $A_{\mu}^{a}$ is described by the equations [10]

$$
\begin{gathered}
\sqrt{p^{2}} \frac{d x^{\mu}}{d \tau}=p^{\mu}, \\
\sqrt{p^{2}} \frac{d p_{\mu}}{d \tau}=Q_{a} F_{\mu \nu}^{a} p^{\nu}, \\
\frac{d Q_{a}}{d \tau}=-f^{a b c} p^{\mu} A_{\mu}^{b} Q_{c},
\end{gathered}
$$

where $\mu=0,1,2,3$ and $f^{a b c}$ are the structure constants of the color group $\mathcal{G}$. It follows from eq.(3) that

$$
\frac{d}{d \tau} Q^{2} \equiv \frac{d}{d \tau}\left(Q_{a} Q_{a}\right)=0
$$

and from eq.(2)

$$
\frac{d}{d \tau} p^{2} \equiv \frac{d}{d \tau}\left(\eta^{\mu \nu} p_{\mu} p_{\nu}\right)=0
$$

where $\eta_{\mu \nu}=(1,-1,-1,-1)$. From eqs.(1) and (5) it follows that

$$
d \tau^{2}=d x^{\mu} d x_{\mu} p^{-2}
$$

Hence, $d \tau$ is $c \sqrt{p^{-2}}$ times the proper time $d t \sqrt{1-c^{-2}\left(\frac{d \mathbf{x}}{d t}\right)^{2}}$ where $x^{0}=c t$ ( we set $c=1$ from now on for convenience).

It will be useful to work with a matrix notation. Let $T_{a}$ be a representation of the algebra of the group $\mathcal{G}$

$$
\left[T_{a}, T_{b}\right]=f^{a b c} T_{c}
$$

normalized by $\operatorname{Tr}\left(T_{a} T_{b}\right)=-\delta_{a b}$. We define $Q=Q_{a} T_{a}$ and $A_{\mu}=T_{a} A_{\mu}^{a}$. Let $A p=p^{\mu} A_{\mu}$ then eq.(3) can be expressed as

$$
\frac{d Q}{d \tau}=-[A p, Q]
$$

It has a solution

$$
Q_{\tau}=U_{\tau} Q_{0} U_{\tau}^{-1}
$$

where $U_{\tau} \in \mathcal{G}$ solves the equation

$$
d U_{\tau}=-p A U_{\tau}
$$


Eq.(7) is realizing the sphere (4) as an orbit of the adjoint representation of $\mathcal{G}$. We shall denote the invariant measure on the sphere $Q^{2} \equiv \operatorname{Tr} Q^{2}$ by $d Q$ (we use capital Latin letters to denote the points on the sphere).

The kinetic theory of classical particles begins with a distribution $\mathcal{K}_{\tau}$ of trajectories $(x(\tau, y, q, Q), p(\tau, y, q, Q), R(\tau, y, q, Q))$ starting from $(y, q, Q)$ in the phase space

$$
\begin{aligned}
& \mathcal{K}_{\tau}(x, p, S ; y, q, Q) \\
& =\delta(x-x(\tau, y, q, Q)) \delta(p-p(\tau, y, q, Q)) \delta(S-R(\tau, y, q, Q)) .
\end{aligned}
$$

We can consider more general distributions of trajectories $\Phi_{\tau}$ by spreading the initial points in the phase space with a certain probability distribution $\Phi$

$$
\Phi_{\tau}(x, p, R)=\int \mathcal{K}_{\tau}(x, p, R ; y, q, Q) \Phi(y, q, Q) d y d q d Q,
$$

( by $d y$ or $d q$ we denote an integral over $R^{4}$ ). $\mathcal{K}_{\tau}$ as well as $\Phi_{\tau}$ satisfy the differential equation

$$
\frac{d}{d \tau} \Phi_{\tau} \equiv \mathcal{L}^{+} \Phi_{\tau}=-\frac{1}{\sqrt{p^{2}}}\left(p^{\mu} \frac{\partial \Phi_{\tau}}{\partial x^{\mu}}+F^{\mu \nu} p_{\nu} \frac{\partial \Phi_{\tau}}{\partial p^{\mu}}+f^{a b c} R_{a} p^{\mu} A_{\mu}^{b} \frac{\partial \Phi_{\tau}}{\partial R_{c}}\right) .
$$

$\mathcal{K}_{\tau}$ solves eq.(10) with the initial condition $\delta(x-y) \delta(p-q) \delta(S-Q)$ and $\Phi_{\tau}(x, p, R)$ with the initial condition $\Phi(x, p, Q)$. In eq.(10) we assumed that $p_{0}$ is an independent variable. Keeping the four-momentum off the mass shell allows to preserve explicit Lorentz invariance. The off-shell formulation appears in the Wigner function approach to quantum theories [9][8][35] (the mass-shell projection is performed as an additional step).

The density of trajectories $\Omega$ in the laboratory time $x^{0}$ can be expressed by $\Phi_{\tau}[9]$

$$
\Omega(x, p, R)=\int d \tau \Phi_{\tau}(x, p, R) .
$$

It satisfies the transport equation

$$
\mathcal{L}^{+} \Omega=0
$$

In the Liouville approach to the classical statistical mechanics we consider functions $W$ on the phase space as observables. We may define the expectation value $\Phi_{\tau}(W)$ of $W$ in the state $\Phi_{\tau}$ as

$$
\begin{aligned}
& \Phi_{\tau}(W)=\left(\Phi_{\tau}, W\right)=\int d x d p d Q \sigma(p) \Phi_{\tau}(x, p, Q) W(x, p, Q) \equiv\left(\Phi, W_{\tau}\right) \\
& =\int d y d q d Q \sigma(q) \Phi(y, q, Q) W(x(\tau, y, q, Q), p(\tau, y, q, Q), R(\tau, y,, p, Q)) .
\end{aligned}
$$

Here, $\sigma(p)$ is a mass distribution depending on $p^{2}$. In order to fix the mass we let $\sigma(p) \rightarrow \delta\left(p^{2}-m^{2}\right)$. The semigroup $\left(T_{\tau} W\right)(y, q, P) \equiv W_{\tau}(y, q, P)=$ 
$W(x(\tau, y, q, P), p(\tau, y, q, P), R(\tau, y, q, P))$ is unitary in the scalar product (13). We have from eq.(13)

$$
\begin{aligned}
& \frac{d}{d \tau} W_{\tau}(y, q, P)=\frac{d}{d \tau}\left(T_{\tau} W\right)(y, q, P)=\left(\mathcal{L} T_{\tau} W\right)(y, q, P) \\
& =\frac{1}{\sqrt{q^{2}}}\left(q^{\mu} \frac{\partial W_{\tau}}{\partial y^{\mu}}+F^{\mu \nu} q_{\nu} \frac{\partial W_{\tau}}{\partial q^{\mu}}+f^{a b c} P_{a} p^{\mu} A_{\mu}^{b} \frac{\partial W_{\tau}}{\partial P_{c}}\right),
\end{aligned}
$$

where the generator $\mathcal{L}$ of the semigroup $T_{\tau}=\exp (\tau \mathcal{L})$ is the adjoint of $\mathcal{L}^{+}$ of eq.(10) in the Hilbert space $L^{2}(\sigma d y d q d P)$. The requirement of the $\tau$ independence of the probability distribution $\left(\frac{d}{d \tau} \Phi_{\tau}=0\right)$ gives the kinetic equation in the laboratory time $t$. This is the same equation as the one which can be derived by an elimination of $\tau$ in favor of $t$ in the evolution equations (1)-(2).

Let us consider the equation (14) for functions $W$ of particle trajectories which is of the form

$$
\partial_{\tau} W=(X+Z+Y) W \equiv(K+Y) W,
$$

where $K=X+Z$. In eq.(15) we have according to eq.(14)

$$
\begin{gathered}
X=p^{\mu} \partial_{\mu}^{x}, \\
Y=-\operatorname{Tr}\left(Q F_{\mu \nu}(x) p^{\nu} \frac{\partial}{\partial p^{\mu}}\right. \\
Z=-\operatorname{Tr}\left(\left[Q, p^{\mu} A_{\mu}(x)\right] \frac{\partial}{\partial Q}\right) .
\end{gathered}
$$

Let

$$
Y(s)=\exp (-s K) Y \exp (s K) .
$$

Then, the solution of eq.(15) can be expressed as (an analog of the interaction picture)

$$
W_{t}=\exp (t K) W_{t}^{I},
$$

where $W_{t}^{I}$ is a solution of the equation

$$
\partial_{s} W_{s}^{I}=Y(s) W_{s}^{I} .
$$

In order to calculate $Y(s)$ we introduce the path-ordered exponential along a curve $\gamma$ starting from $x$

$$
U_{\gamma y}=P\left(\exp \left(-\int A_{\mu} d \gamma^{\mu}\right)\right) .
$$

with $y$ as the endpoint of the curve. It is a solution of the equation

$$
\frac{\partial}{\partial y^{\mu}} U_{\gamma y}=-A_{\mu}(y) U_{\gamma y} .
$$

Under the gauge transformation

$$
A_{\mu} \rightarrow g A_{\mu} g^{-1}-g \partial_{\mu} g^{-1}
$$


we have $F_{\mu \nu}(y) \rightarrow g(y) F_{\mu \nu}(y) g(y)^{-1}$ and

$$
U_{\gamma y} \rightarrow g(y) U_{\gamma y} g^{-1}(x)
$$

In terms of the path ordered exponential the solution of eq.(3) is

$$
Q(\tau)=U_{p}(\tau) Q(0) U_{p}^{-1}(\tau),
$$

where $U_{p}(\tau)=U_{\gamma y}$ is calculated along the straight line in the direction $p$,i.e., $\gamma(s)=x-p s$ with $y=x-p \tau$.

We define the corresponding gauge covariant field strength transported along the straight line [32][33]

$$
\hat{F}_{\mu \nu}(p, x-p s)=U_{p}(s)^{-1} F_{\mu \nu}(x-p s) U_{p}(s) .
$$

Under a gauge transformation

$$
\hat{F}_{\mu \nu}(p, x-p s) \rightarrow g(x) \hat{F}_{\mu \nu}(p, x-p s) g(x)^{-1}
$$

(here $x$ is a fixed point, so the gauge rotation does not change with $s$ ). From eqs.(17) and (19) (using the Hausdorf-Baker formula) we obtain

$$
\begin{aligned}
& Y(s)=-\operatorname{Tr}\left(Q(s) F_{\mu \nu}(x-s p)\right) p^{\nu}\left(\frac{\partial}{\partial p_{\mu}}+s \frac{\partial}{\partial x_{\mu}}\right)+s f^{a b c} Q_{a} A_{\rho}^{b} Q_{d} F_{d}^{\sigma \rho} p_{\sigma} \frac{\partial}{\partial Q_{c}}+o\left(s^{2}\right) \\
& =-\operatorname{Tr}\left(Q(0) \hat{F}_{\mu \nu}(p, x-s p)\right) p^{\nu}\left(\frac{\partial}{\partial p_{\mu}}+s \frac{\partial}{\partial x_{\mu}}\right)+s f^{a b c} Q_{a} A_{\rho}^{b} Q_{d} F_{d}^{\sigma \rho} p_{\sigma} \frac{\partial}{\partial Q_{c}}+o\left(s^{2}\right) .
\end{aligned}
$$

The terms linear in $s$ as well as the $o\left(s^{2}\right)$ terms (higher orders in $s$ ) will be irrelevant for the calculation of the diffusion performed in the next section.

The assumption that the field strength $F$ is constructed from $A_{\mu}$ is expressed as the Bianchi identities

$$
\epsilon^{\mu \nu \sigma \rho} D_{\mu} F_{\sigma \rho}=0,
$$

where $D_{\mu} F_{\sigma \rho}=\partial_{\mu} F_{\sigma \rho}+\left[A_{\mu}, F_{\sigma \rho}\right]$. We consider random gluon fields as an approximation to quantum fields. In general, correlation functions of gauge fields being gauge dependent are not well-defined. We are going to define a two-point correlation function of gauge covariant variables

$$
\hat{F}_{\mu \nu}(\gamma, y)=U_{\gamma y}^{-1} F_{\mu \nu}(y) U_{\gamma y} .
$$

$\hat{F}$ satisfies the Bianchi identities with an ordinary derivative (instead of the covariant one;this has been shown first in [32], see also [33])

$$
\epsilon^{\mu \nu \sigma \rho} \partial_{\mu} \hat{F}_{\sigma \rho}=0
$$

We treat the Wong equations (1)-(3) in a random Yang-Mills field as an approximate description of the dynamics of quarks in quantum gauge theory 
at high temperature. Then, the expectation value of an observable $\mathcal{O}$ at the inverse temperature $\beta$ is defined as

$$
\langle\mathcal{O}\rangle_{\beta}=\operatorname{Tr}\left(\exp \left(-\beta w_{\mu} P^{\mu}\right) \mathcal{O}\right)
$$

where $P^{\mu}$ is the generator of space-time translations and the four-vector $w^{\mu}$ $\left(w^{\mu} w_{\mu}=1\right)$ describes the reference frame [37].

In QED (and perturbative QCD) the expectation value (30) leads to the distribution $n(p ; w)$ of photons (gluons) according to the covariant Bose-Einstein law

$$
n(p ; w)=\left(\exp \left(\beta w^{\nu} p_{\nu}\right)-1\right)^{-1}
$$

\section{Diffusive motion in a random gauge field}

The two-point correlation functions of gauge covariant variables $\hat{F}(p, x-p s)$ are covariant under transformations of the Poincare group (acting upon $x, p$ and $w)$ and invariant under transformations of the (color) $\mathcal{G}$ symmetry. We assume that the non-commutativity of quantum gauge fields can be neglected at high temperature in the calculation of the correlation functions (this can be shown in perturbation theory in the state (30)). Then, the symmetry properties with respect to an exchange of indices together with the Bianchi identities (29) lead to the representation ( the Bianchi identities in the form (29) allow to apply the same methods for the two-point function as in the Abelian case of [36])

$$
\left.\left\langle\hat{F}_{\mu \nu}^{a}(p, x-p s)\right) \hat{F}_{\sigma \rho}^{b}\left(p, x-p s^{\prime}\right)\right\rangle \equiv \delta^{a b} G_{\mu \nu ; \sigma \rho}^{p}\left(p\left(s^{\prime}-s\right) ; s, s^{\prime}, w\right),
$$

where

$$
\begin{aligned}
& G_{\mu \nu ; \sigma \rho}^{p}\left(y ; s, s^{\prime}, w\right) \\
& =\int d k \tilde{G}\left(s, s^{\prime}, k, p, w\right)\left(\eta_{\mu \sigma} k_{\nu} k_{\rho}-\eta_{\mu \rho} k_{\nu} k_{\rho}+\eta_{\nu \rho} k_{\sigma} k_{\mu}-\eta_{\nu \sigma} k_{\mu} k_{\rho}\right) \exp (i k y) .
\end{aligned}
$$

$\tilde{G}$ is a Lorentz invariant function of $k, p$ and $w$. Let us note that

$$
G_{\mu \nu ; \sigma \rho}^{p}(0 ; 0,0, w)=\eta_{\mu \sigma} T_{\nu \rho}-\eta_{\mu \rho} T_{\nu \sigma}+\eta_{\nu \rho} T_{\sigma \mu}-\eta_{\nu \sigma} T_{\mu \rho}
$$

where

$$
T_{\mu \nu}=\int d k \tilde{G}(0,0, k, 0, w) k_{\mu} k_{\nu}=\rho w_{\mu} w_{\nu}-\pi_{E}\left(\eta_{\mu \nu}-w_{\mu} w_{\nu}\right)
$$

Eq.(35) gives a decomposition of the tensor $T_{\mu \nu}$ in terms of $\eta_{\mu \nu}$ and $w_{\mu} w_{\nu}$. If $\tilde{G}$ is non-negative then $\rho$ and $\pi_{E}$ must be non-negative. $\tilde{G}$ at $s=s^{\prime}=0$ does not depend on $p$ because $p$ can enter $F$ only through the path starting in 0 
and ending in $s p . T_{\mu \nu}$ is related to the expectation value (32) of the Yang-Mills energy-momentum tensor. In order to see this let us calculate

$$
-T r\left\langle F_{\mu \rho} F_{\nu \sigma} \eta^{\rho \sigma}-\frac{1}{4} \eta_{\mu \nu} F_{\sigma \rho} F^{\sigma \rho}\right\rangle=2 T_{\mu \nu}-\frac{1}{2} \eta_{\mu \nu} T_{\rho}^{\rho}
$$

We are going to approximate the evolution (15) by a diffusion in momentum space. As follows from eqs.(1)-(3) such an evolution is covariant but not invariant with respect to the gauge transformations. We show that the diffusion in the momentum space can be defined in a gauge invariant way. The color also evolves in time. However, this evolution is gauge-dependent. We give arguments at the end of this section that if the color evolution is approximated by diffusion then there is a gauge such that the color diffusion constant is equal to zero.

We apply the approach of Kubo [20]-[21] which approximates the random Liouville operator on the rhs of eq.(10) by the expectation values of $Y$ and its square

$$
\left\langle W_{\tau}^{I}\right\rangle \simeq \exp \left(\int_{0}^{\tau} d s\langle Y(s)\rangle+\frac{1}{2}\left\langle\left(\int_{0}^{\tau} d s \tilde{Y}(s)\right)^{2}\right\rangle\right) W
$$

where

$$
\tilde{Y}=Y-\langle Y\rangle
$$

The second order term resulting from the square of $Y$ reads ( we omit the terms with the derivatives over the charges $Q$ restricting ourselves to the diffusion in momentum; we assume that the initial state $W$ in eq.(15) does not depend on Q)

$$
\begin{aligned}
& \lim _{\tau \rightarrow 0} \frac{1}{\tau^{2}}\left\langle\left(\int_{0}^{\tau} Y(s) d s\right)^{2}\right\rangle=\lim _{\tau \rightarrow 0} \frac{1}{2 \tau^{2}} \int_{0}^{\tau} d s \int_{0}^{\tau} d s^{\prime}\left\langle Y(s) Y\left(s^{\prime}\right)+Y\left(s^{\prime}\right) Y(s)\right\rangle \\
& =p^{-2} Q^{2}\left(\eta_{\mu \sigma} T_{\nu \rho}-\eta_{\mu \rho} T_{\nu \rho}+\eta_{\nu \rho} T_{\sigma \mu}-\eta_{\nu \sigma} T_{\mu \rho}\right) p^{\nu} \partial^{\mu} p^{\rho} \partial^{\sigma} \equiv-\mathcal{A}_{w} .
\end{aligned}
$$

Elementary calculations using eq.(34) give

$$
\begin{aligned}
& \mathcal{A}_{w}=-2 \pi_{E} Q^{2} \partial_{\mu} P^{\mu \nu} \partial_{\nu}+Q^{2}\left(\rho+\pi_{E}\right) p^{-2}\left((w p)^{2} \partial_{\mu} \partial^{\mu}-2 w p w^{\mu} p^{\rho} \partial_{\mu} \partial_{\rho}\right. \\
& \left.+p^{2} w^{\mu} w^{\rho} \partial_{\mu} \partial_{\rho}-w^{2} p^{\nu} \partial_{\nu}-2 w p w^{\nu} \partial_{\nu}\right) \equiv \partial_{\mu} \alpha^{\mu \nu} \partial_{\nu},
\end{aligned}
$$

where

$$
P^{\mu \nu}=\eta^{\mu \nu}-p^{-2} p^{\mu} p^{\nu}
$$

and $Q^{2} \equiv \operatorname{Tr} Q^{2}=Q_{a}(\tau) Q_{a}(\tau)=Q_{a}(0) Q_{a}(0)$ is the square of the charge (which is a constant according to eq.(7)). The term $s \partial_{\mu}^{x}$ of eq.(27) as well as the remaining terms linear in $s$ and $o\left(s^{2}\right)$ do not contribute to $\mathcal{A}_{w}$.This is so because it is of higher (third) order in $\tau$. The limit in eq.(40) depends only on $G(0)$ corresponding to $s=s^{\prime}=0$ because taking into account $s$ in $G$ would give higher orders in $\tau$. As a consequence the $T_{\mu \nu}$ coefficients depend neither on $x$ nor on $p$ because the dependence on $p$ is always multiplied by $s$. According to Kubo [20]-[21] the $\tau^{2}$ behavior at short time (small in comparison to the correlation time $\tau_{c}$ ) of the random evolution goes over to the linear behavior at long time with the generator $\mathcal{A}_{w}$ multiplied by the correlation time $\tau_{c}$ of the gluon modes. 
In order to justify a replacement of the Liouville evolution (15) by the diffusion (40) at an arbitrarily long time we have to apply the Markov approximation. In such a case the dynamics is viewed as a composition of independent short time evolutions starting anew after the correlation time $\tau_{c}$ (see the discussion in Kubo [20]-[21] ). The rigorous proof [22] requires a proper scaling of fields and the time in order to determine precisely the conditions required for the limiting diffusive behavior.

We define the diffusion generator by the lowest order $\left(\tau^{2}\right)$ term in the expansion (37) in proper time. We incorporate Kubo's argument that this operator multiplied by the correlation time generates a diffusion properly approximating the random dynamics. In this way we are led to the following diffusion equation for particles with a continuous mass spectrum ( by a certain abuse of notation we denote by $W$ the expression which has the meaning of $\langle W\rangle$ in random dynamics of sec.2)

$$
\frac{1}{\tau_{c}} \partial_{\tau} W=\frac{1}{\tau_{c}} p^{\mu} \partial_{\mu}^{x} W+\partial_{\mu} \alpha^{\mu \nu} \partial_{\nu} W
$$

The diffusion in the proper time originates from the relativistic dynamics in the proper time. Then, the proper time has a well-defined meaning. This is the time measured in the frame moving with the particle. However, if the particle motion is random then this frame is random as well. Such a random frame is loosing a physical meaning. For this reason we should express the kinetic transport in terms of the coordinate (laboratory) time. The probability distribution evolves according to the adjoint equation (10). The independence of the proper time parametrization $\left(\frac{d}{d \tau} \Phi_{\tau}=0\right)$ leading to the transport equation (12) is equivalent to the replacement of the proper time by the physical time $t$ in the kinetic equation (12). We extend this requirement to the diffusion (41). In this way we obtain the transport equation

$$
\begin{aligned}
& \frac{1}{\tau_{c}} p^{\mu} \partial_{\mu}^{x} \Omega=\partial_{\mu} \alpha^{\mu \nu} \partial_{\nu} \\
& =Q^{2} \partial_{\mu}\left(2 \pi_{E} P^{\mu \rho} \partial_{\rho}-\left(\rho+\pi_{E}\right) p^{-2}\left((p w)^{2} \partial^{\mu}-(w p)\left(w^{\mu} p^{\rho}+w^{\rho} p^{\mu}\right) \partial_{\rho}+p^{2} w^{\mu} w^{\rho} \partial_{\rho}\right) \Omega .\right.
\end{aligned}
$$

We have shown [36] that the positivity condition

$$
a_{\mu} a_{\nu} \alpha^{\mu \nu} \geq 0
$$

is satisfied under the condition

$$
\rho \geq \pi_{E} \geq 0
$$

We can decompose the diffusion (42) into diffusions on the mass shell using the formula

$$
\sigma(p)=\int d m^{2} \sigma\left(m^{2}\right) \delta\left(p^{2}-m^{2}\right)
$$


Then, at zero temperature (realized by setting $\pi_{E}+\rho=0$ in eq.(42)) we have

$$
-\int d p \sigma(p) \Phi \partial_{\mu} p^{2} P^{\mu \nu} \partial_{\nu} W=\int d p d m^{2} \sigma\left(m^{2}\right) \delta\left(p^{2}-m^{2}\right) \Phi \triangle_{H}^{m} W
$$

where

$$
\triangle_{H}^{m}=\left(\delta^{j l} m^{2}+p^{j} p^{l}\right) \frac{\partial}{\partial p^{l}} \frac{\partial}{\partial p^{j}}+3 p^{l} \frac{\partial}{\partial p^{l}}
$$

$j, l=1,2,3$. and $\triangle_{H}^{m}$ is the generator of the relativistic diffusion defined by Schay[27] and Dudley [28].The relativistic invariance is fixing the diffusion coefficients in front of the $\delta^{j l}$ and $p^{j} p^{l}$ terms which remained undetermined in [4] and $[15][16]$.

We did not discuss the charge evolution assuming that the initial state $W$ does not depend on the charge $Q$. We could apply the diffusion approximation to the random charge evolution determined by the fluctuations of $A_{\mu}$ in eq.(3). If we choose the coordinate gauge [38] $y^{\mu} A_{\mu}(x+y)=0$ then

$$
A_{\mu}^{a}(x-p s)=\int_{0}^{1} d \lambda F_{\mu \nu}^{a}(x-\lambda s p) s p^{\nu} .
$$

The diffusion constant evaluated from $\left\langle\frac{d Q^{a}}{d \tau} \frac{d Q^{b}}{d \tau^{\prime}}\right\rangle$ (using eq.(3)) along the straight line $y=s p$ is zero. This calculation indicates that the color diffusion calculated in [15]-[16] may be a spurious phenomenon.

The diffusion (42) determines the phase space distribution W. In terms of $W$ we can define a velocity of the fluid of diffusing particles and its energymomentum tensor. Such an approach leads to a hydrodynamic description [39] resulting directly from QCD (in the Wong approximation).

\section{Summary}

We have formulated the problem of a transport theory of quark-gluon plasma starting from quantum gauge theory at finite temperature in semiclassical approximation which is applicable at high temperature and low momenta. In this approximation the quarks are treated as classical color particles and gauge fields as classical random fields. In this paper we have shown that the diffusion in momentum results from a model of a particle moving in a gauge field in the same way as a non-relativistic diffusion results from an evolution in a random electric and magnetic fields [18] [19]. The Wong equation has the gauge coupling constant as the only parameter. Then, we derive the diffusion equation which depends on two parameters $\rho$ and $\pi_{E}$ which could be calculated from the correlation functions in the Euclidean framework on the lattice (the diffusion constant has been expressed by correlation functions of Wilson loops in finite temperature QCD in an earlier paper [17]). The diffusion equation could be directly compared with experimental data ( see ref. [5]) or we could use it for 
some other approximations. We have in particular in mind the hydrodynamic equations which could be derived from the diffusion equation. The parameters entering the fluid equations could be expressed by the ones of the diffusion equation.

\section{References}

[1] E.M. Lifshits and L.P. Pitaevskij, Physical Kinetics,Pergamon Press,1981

[2] R.C. Hwa,Phys.Rev.D32,637(1985)

[3] B.Svetitsky, Phys.Rev.D37,2484(1988)

[4] D.B. Walton and J. Rafelski,Phys.Rev.Lett.84,31(2000)

[5] R.Rapp and H. van Hees,in Quark-Gluon Plasma 4, ed.by R.C.Hwa and Xin-Nian Wang,World Scientific,2010; arXiv:0903.10961

[6] U. Heinz, Phys.Rev.Lett.51,351(1983)

[7] U. Heinz, Ann.Phys.161,48(1985)

[8] H.-Th. Elze and U. Heinz, Phys.Rep.183,81(1989)

[9] S.R. de Groot, W.A. van Leeuwen and Ch.G. van Weert, Relativistic Kinetic Theory, North Holland,1980

[10] S.K. Wong, Nuovo Cimento,A65,689(1975)

[11] L.S. Brown and W.I. Weisberger, Nucl.Phys.B157,285(1979)

[12] J. Frenkel and J.C. Taylor, Nucl.Phys.B334,199(1990)

[13] P.F. Kelly,Q.Liu, C. Lucchesi and C. Manuel, Phys.Rev.D50,4209(1994)

[14] D.F. Litim and C. Manuel,Phys.Rep.364,451(2002)

[15] A.Selikhov and M. Gyulassy, Phys.Lett.B316,373(1993)

[16] A.Selikhov and M. Gyulassy, Phys.Rev.C49,1726(1994)

[17] J. Casalderrey-Solana and D.Teaney, Phys.Rev.D74,085012(2006)

[18] W.B. Thompson and J. Hubbard, Rev.Mod.Phys.32,714(1960)

[19] S. Ichimaru, Basic Principles of Plasma Physics, Benjamin-Cummings, London, 1973

[20] R.Kubo, J.Math.Phys.4,174(1962) 
[21] R. Kubo, M.Toda and N.Hashitsume, Statistical Physics II.Nonequilibrium Statistical Mechanics, Springer, Berlin,1985

[22] H. Kesten and G.C. Papanicolaou, Commun.Math.Phys.78,19(1980)

[23] J. Dunkel and P. Hänggi,Phys.Rep.471,1(2009)

[24] C. Chevalier and F. Debbasch, AIP Conf.Proc.913,42(2007)

[25] J. Lopuszanski, Acta Phys.Polon.12, 87(1953)

[26] R. Hakim, J.Math.Phys.9,1805(1968)

[27] G.Schay,PhD thesis,Princeton University,1961

[28] R.Dudley, Arkiv for Matematik,6,241(1965)

[29] Z. Haba, Phys.Rev.E79,021128(2009)

[30] J.A. Alcantara and S. Calogero, Kinetic and Related Models, 4,401(2011)

[31] C. Chevalier and F. Debbasch, J.Math.Phys.49,043303(2008)

[32] I. Bialynicki-Birula, Bull.Acad.Polon.Sci.11,135(1963)

[33] S. Mandelstam, Phys.Rev.175,1580(1968)

[34] Z. Haba, Journ.Phys.A44,335202(2011)

[35] S.Mrowczynski, Ann.Phys.(N.Y.)169,48(1986)

[36] Z. Haba, Journ.Phys.A46,155001(2013)

[37] T. Matsui, B. Svetitsky and L.D. McLerran, Phys.Rev.D34,783(1986)

[38] L. Durand and E. Mendel, Phys.Rev.D26,1368(1982)

[39] P. Romatschke, ArXiv:0902.3663 